\title{
Near patient testing and pathology in the new millennium
}

\author{
M A Crook
}

Recently laboratories have tended to become more specialised and also centralised. Some process millions of samples a year, somewhat like a factory assembly line. High throughput, cost-effective automation (perhaps including the use of robotics), stringent quality assurance processes, computerisation with data storage and retrieval systems, and highly skilled monitored personnel are now common practice in many laboratories. Paradoxically, at the same time there has been a gradual growth in the decentralisation of laboratory tests for patient management, with test analysis being performed at the bedside, clinic, or by the patients themselves at home. This process has been broadly called "near patient testing" or NPT. Many terms have been ascribed to laboratory testing in sites other than centralised laboratories such as: alternative site testing, point of care testing, physician office laboratory testing, bedside testing, limited service laboratory testing, ancillary testing, out of laboratory testing, and near patient testing, to name but a few. However, in the United Kingdom NPT is popularly used, although the question arises of how near is near, and what about individuals who are not patients? The term "point of care testing" may be preferable, as this would include bedside testing, in vivo testing, physician office laboratory testing, and patient self testing. ${ }^{1}$

In a classic paper about 15 years ago, the basic philosophy behind NPT in the context of clinical biochemistry was described. ${ }^{2}$ New technologies now exist that have brought into being this prophetic message. Indeed, matters have now extended beyond clinical biochemistry to other pathology disciplines, including microbiology and haematology. This recent interest in NPT returns us to over 100 years ago when clinicians would taste their patients' urine at the bedside to help diagnosis diabetes mellitus, or would evaluate patients' body fluids with a microscope on the ward.

\section{Advantages of NPT}

TURNAROUND TIMES

One of the main advantages of NPT over laboratory testing is the relative immediacy of results. Some clinicians feel that this would enable them to instigate treatment quickly and shorten the wait for the patient. It may also reduce the number of outpatient appointments and clinic visits for the patient.

Several of the NPT devices require minimal specimen preparation or collection (in some cases using a finger prick specimen of blood). Furthermore, the analysis time is often relatively short and by definition the machine is near the patient, thus shortening the transport time of specimens and reports.

When considering result turnaround time, it is useful to consider the concept of the "total testing cycle." This is a loop starting with the clinician requesting a test which leads to the specimen being collected and arriving at the place of analysis, followed ultimately by analysis and result transmission back to the requesting clinician. NPT allows the turnaround time to be shortened, mainly by shortening the preand postanalytical steps.

\section{TECHNOLOGY ADVANCES AND EASE OF USE OF NPT}

The recent increase in NPT has partly reflected technological change in the design of analysers. With the advent of microchips, computerisation, and miniaturisation, it has become much easier to bring analyses nearer to the patient and for them to be performed by less trained personnel or by the patients themselves. Some of the modern NPT devices incorporate biosensors, electrodes, and dry and solid phase chemistry reagents. In the case of solid phase chemistry sample separation devices and analytical reagents are incorporated into a single assay element and the reaction chemistries are often followed by reflectance photometry or front face fluorescence. These can allow small sample and reagent volumes, quick assay reaction times, ease of use and disposal of used reagents, more than one analyte to be measured simultaneously, and probably less technical skill.

Mention should also be made of continuous non-invasive monitoring. Transcutaneous biosensors have been developed that allow continuous measurements to be made through the patient's skin without the need for blood collection. Consider also near-infrared spectroscopy which may allow continuous monitoring of more than one analyte, and also in vivo glucose monitoring with implantable sensors in diabetic patients. ${ }^{3}$

Depending upon the technology used there may be greater test flexibility in NPT analysis, rather than being "locked in" to laboratory analyser test profiles. In choosing an NPT analyser system it is important to remember that duplication may occur within the hospital at separate sites. Furthermore, different analysers on the same site may have different reference ranges and this may create difficulties in comparing results. Sometimes, demarcation disputes arise when a machine fails and issues of backup facilities result. Many of the new NPT analysers are relatively easy to maintain but maintenance may need to be carried out by non-laboratory staff out of the laboratory 
setting. It is also likely that results will need to be interpreted and trouble shooting performed by non-laboratory personnel.

costs

The reduction in turnaround time may result in a reduction in total costs if episodes of illness are shortened and transport costs reduced, for example courier costs. However, on a direct charge basis including capital costs, NPT maybe more expensive than central laboratory testing, reflecting duplication of tests and the economies of scale that result from centralised laboratory testing. Reagent and machine costs, quality control material costs, maintenance costs, report forms and results storage costs, and training costs may all need to be taken into consideration when embarking upon NPT. Labour costs are more difficult to assess in NPT but may incorporate nursing staff; however, set against this are possible savings of on-call or out of hours costs of laboratory staff.

It may be difficult to ascertain the cost of an NPT program, which may be very institution dependent. There is a tendency to underestimate resources needed for such a program; it has been suggested that the cost of glucose NPT is between three and four times that of the main laboratory. ${ }^{4}$ However, depending upon the structure and organisation of the NPT setting, the overall costs could be less when the merits of rapid therapeutic response and shorter hospital stay are taken into consideration (table 1 ).

\section{Some possible clinical settings for NPT devices}

ACCIDENT AND EMERGENCY

One of the potential advantages of NPT is the possibility of a quick turnaround time for a result, and this is particularly important in the accident and emergency (A\&E) department. A significant number of A\&E departments have blood gas machines, blood glucose meters, and even small analysers for common biochemical tests. One particular area in A\&E where NPT is rapidly taking hold is in the biochemical diagnosis of acute myocardial infarction. The introduction of thrombolytic treatment for myocardial infarction has been shown to improve survival but ideally it should be instigated as soon as possible-hence the interest in NPT. Troponin T or I have high cardiac specificity. For example, a whole blood troponin T NPT device is available; one of the first marketed in the United Kingdom was the Boehringer-Mannheim TROP T rapid assay. ${ }^{5}$

Another potential use of NPT in A\&E is in drug overdoses. The AcetaSite test card with Stat-Site reflectance meter is a bedside method for measuring plasma paracetamol concentrations that takes a few minutes for a result. ${ }^{6}$

The recently developed Hewlett-Packard I-Stat system is called by the manufacturers a "point of care blood analysis system." This novel system uses cartridges that are filled with whole fresh blood (two to three drops) and which are then inserted into a hand held analyser which gives results within about two minutes. The hand held analyser is battery
Table 1 Some possible advantages and disadvantages of near patient testing (NPT)

Advantages

NPT methods may be less clinically invasive, for example finger prick of blood or biosensor

Possible on-line monitoring for patients with NPT

NPT may be advantageous in remote areas

Patients can be more involved in their care with NPT

Greater laboratory test selectivity as there is less requirement to be restricted to analyser dedicated "profiles" when using NPT

Usually shorter result turnaround time for NPT and thus earlier treatment modification or initiation

Possibly reduced on call staff costs, but institution dependent NPT may save sample transport and reporting costs

Disadvantages

NPT tests may be performed by non-laboratory-trained staff Reference ranges and results may differ from laboratory, thus making comparisons difficult

Care needed in NPT machine maintenance and repairs Importance of adequate NPT quality control and assurance Possible problems of who will interpret test result

Possible lack of backup support should NPT analyser fail

Duplication of equipment possible in different hospital sites

The ready availability of tests may cause increased

inappropriate testing

Without economies of scale tests may become expensive

powered and can download results into a computer database if required. The manufacturer claims very short turnaround times, as sample preparation and transport are minimal. Various different test cartridges are available for different analytes, including electrolytes, urea, glucose, ionised calcium, blood gases, and packed cell volume. An important study examined the use of the I-Stat system in the A\&E setting. ${ }^{7}$ The machine reduced the waiting time for a result in comparison with the hospital laboratory, and hence also the time to embark upon appropriate clinical management. However, the patients did not spend less time in the casualty department, nor did the use of the system necessarily affect clinical outcome.

\section{COAGULATION CLINICS}

Various devices are available for measuring prothrombin or activated partial thrombin time (APTT), using capillary or venous blood. These can be used in coagulation clinics or by patients on warfarin to monitor their own treatment.

\section{DRUG ADDICTION CLINICS}

The ability to measure misused drugs and ethanol is of interest in drug and ethanol addiction clinics. Indeed, this has extended to the workplace to screen workers for substance abuse. Various systems are available including the Boehringer-Mannheim Frontline range. This is essentially a urine dipstick method that can give a semiquantitative drug result within a few minutes. The method uses a GLORIA technique (gold labelled, optically read, rapid immuno assay). Drugs that can be measured now or in the near future include opiates, cocaine, cannabis, benzodiazepines, amphetamines, and methadone. Another system is the Ontrak range from Roche Diagnostic Systems, which also uses urine and gives a positive or negative result. Unlike the Frontline method, urine is mixed with reagents in a kit and after mixing a milky appearance gives a positive result. Several drugs can be tested (each need- 
ing a different slide, as for the Frontline reagent strips), including amphetamine, barbiturates, benzodiazepines, cocaine, and morphine. Roche Diagnostics also have a similar system called Onsite, for qualitative testing for ethanol in either saliva or urine. Individuals may be able to test themselves for ethanol, particularly in the context of drinking before driving.

GENERAL PRACTICE, OUTPATIENT CLINICS, AND WARDS

Many outpatient clinics, wards, and general practices use urine dipstick testing for screening patients. Various urine testing strips are available. Two of the most popular ranges in the United Kingdom are those marketed by Boehringer-Mannheim (BM-test strip range) and Bayer's Multistix reagent strips. Some of these tests can be useful to screen for urinary tract infections. There are various machines to determine cholesterol, triglyceride, and HDL cholesterol. One is the Boehringer-Mannheim Reflotron 2000 machine and another is the Cholestech L-D-X analyser, which may be useful in general practice surgeries or lipid clinics for cardiovascular risk screening. Some desk top analysers also allow other variables to be assayed, for example creatinine, glucose, bilirubin, and haemoglobin. ${ }^{8}$

NEONATAL CARE AND ADULT INTENSIVE CARE One test of particular relevance for neonatal units is the determination of blood bilirubin using NPT bilirubinometers. In addition, blood gas machines and mini analysers such as the Hewlett-Packard I-Stat can offer a comprehensive repertoire of biochemical and haematological tests in these units.

PATIENT SELF-TESTING

Pregnancy self-testing is one of the most common forms of NPT. Over-the-counter pregnancy test kits have recently been evaluated by the Medical Devices Agency (MDA).

Testing for faecal occult blood as a diagnostic aid for colorectal carcinoma is another selftesting possibility. Strictly speaking, the individual is not yet a "patient," but a participant in a screening programme. One of the most thoroughly evaluated methods is the Haemoccult (trade mark of SmithKline-Beckman Corporation, USA). This works on a guaic method for detecting faecal occult blood and apparently has a high specificity for colorectal carcinoma and acceptable sensitivity. The test has the advantage of being simple, hygienic, and disposable. The design involves the use of a slide and does not require direct contact with the faeces. It takes only a few minutes to obtain a result and refrigeration is not required. Two randomised trials using this method in screening for colorectal carcinoma have been published, showing its potential in reducing the mortality of colorectal carcinoma. ${ }^{9} 10$

NEAR PATIENT TESTING AND DIABETES MELLITUS NPT self monitoring is often used in the management of diabetes mellitus, involving urine glucose determinations, tests for ketones in
Table 2 Clinical situations in diabetes mellitus where NPT blood glucose assay may be of benefit

When instigating a new treatment regime Pregnancy

Brittle or unstable diabetes

Frequent hypoglycaemic episodes, particularly if no warning

Home management of intercurrent illness

If urine glucose testing has been shown to be inadequate

Stabilisation of patient on insulin as an outpatient

Outreach clinics or when managing patient far away from main laboratory

To help assess day to day glycaemic control in presence of abnormal glycated haemoglobin

When driving or engaged in strenuous activities

urine or plasma, blood glucose measurements, glycated haemoglobin assays, and urinary microalbumin tests. ${ }^{5}$

It is important to note that NPT in diabetes mellitus is performed at enormous cost to the country. In 1995 over $£ 42$ million was spent on glucose home monitoring alone in the United Kingdom. It has been argued that glucose self monitoring should only be performed in circumstances where it serves a definite purpose, as in some cases harm can result. ${ }^{11}$ Table 2 lists the situations in which NPT blood glucose monitoring may be beneficial in diabetes mellitus.

Getting started with near patient testing There is a potential minefield for the novice attempting NPT. Though NPT involves the use of simple to operate machines and straightforward techniques, this does not mean that errors do not occur. The Guidelines for implementation of near patient testing prepared by a working party of scientific members of the Association of Clinical Biochemists (ACB), along with corporate membership of the ACB and representation from the Royal College of Pathologists, are useful and should be consulted. These guidelines have the support of the Royal College of Pathologists and Royal College of General Practitioners. ${ }^{12}$ The Medical Device Directorate (MDD) of the Department of Health publishes evaluations of new equipment which may be relevant (MDD, 14 Russell Square, London WC1 5EH). The Task Force for Alternate Site Testing of the College of American Pathologists in 1994 published a position statement which is also pertinent. When deciding upon a possible NPT system, it may be useful to consider the features shown in table 3. These broad points have been summarised in a recent letter to the British Medical fournal suggesting that minimum standards should be set for near patient testing. ${ }^{13}$ These are as follows and are derived from various sources $^{14-20}$ :

1. There should be a formal training programme for those personnel performing NPT.

2. The users of the NPT apparatus should display competence at regular intervals.

3. NPT should only be performed by certified users.

4. A quality assurance programme, including both internal and external quality control, should be operational, preferably involving the local pathology laboratory. 
Table 3 Factors that may influence the choice of a near patient device

How are samples prepared and how much is needed, for example whole blood or serum?

Is machine easy to use and what are the staff skill requirements?

What size is the machine and how portable?

Is machine reliable, safe, and robust?

Can machine cope with workload and tests required?

Machine accuracy (how close are results to "true" or definitive method?)

Precision (how repeatable is result)

Minimum detection limit (what is lowest detectable concentration?)

Response time and warm up time (how quick is result)

Is there machine result drift during assay?

Is there a wide analytical range or linearity?

What are interfering compounds?

Is machine temperature dependent?

How easy is machine calibration?

Is a staff training programme included?

How are reagents stored?

How long is the operating lifetime?

Is maintenance easy and is a contract included or is this extra? Easy to decontaminate?

Cost of machine, reagents, calibration and quality control

Is assay comparable to those of local laboratory?

Is a quality control scheme included?

Will machine soon become obsolete?

5. Well defined user manuals, including standard operating procedures, should be kept near the apparatus.

6. Apparatus and associated equipment should be adequately maintained and cleaned on a regular basis.

7. Adequate documentation of results and an equipment log book should be kept.

8. The laboratory may be able to advise about what equipment to purchase.

The above list is not necessarily exhaustive but would form the basis of a minimum standard of expectation.

\section{Conclusions}

In summary, we see that there are many possibilities for NPT and its use will undoubtedly expand in the new millennium. Individuals will have greater access to NPT devices and presumably will be increasingly performing such tests upon themselves. However, the tests need to be performed correctly and thus minimum standards of NPT provision are necessary.

1 Handorf CR. Quality control and quality management of alternate site testing. Clin Lab Med 1994;14:539-57.

2 Marks V. Clinical biochemistry nearer the patient. BMf 1983;286:1166-7.

3 Marks V, Alberti KGMM. Clinical biochemistry nearer the patient. Edinburgh: Churchill Livingstone, 1985.

4 Greendyke RM. Cost-analysis-bedside blood glucose testing. Am $\mathcal{F}$ Clin Pathol 1992;97:106-7.

5 Crook M. Handbook of near patient testing. London: Greenwich Medical Media, 1999.

6 Egleston CV, Browning C, Hamdi I, et al. Comparison of two assays for measuring plasma concentrations of paracetamol. BMF 1997;315:991-2.

7 Kendall J, Reeves B, Clancy M. Point of care testing: randomised controlled trial of clinical outcome. BMF 1998:316:1052-7.

8 Hobbs FDR, Broughton PMG, Kenkre JE, et al. Comparison of the use of four desk-top analysers in six urban general practices. Br f Gen Pract 1992;42:317-21.

9 Kronberg O, Fenger C, Olsen J, et al. Randomised study of screening for colorectal cancer with faecal-occult blood test. Lancet 1996;348:1467-71.

10 Hardcastle JD, Chamberlain J, Robinson MHE, et al. Randomised controlled trial of faecal-occult blood screening for colorectal cancer. Lancet 1996;348:1472-7.

11 Gallichan $M$. Self monitoring of glucose by people with diabetes. BMF 1997;314:964-6.

12 Rink R, Hilton S, Szczepur A. Impact of introducing near-patient testing for standard investigations in general practice. BMF 1993;307:775-8.

13 Crook M. Minimal standards should be set for near patient testing. BMF 1996;312:1157.

14 Marks V. Essential considerations in the provision of near patient testing facilities. Ann Clin Biochem 1988;25:220-5.

15 Evans SJ, McVittie JD, Kay JDS. The use of diagnostic equipment outside the diagnostic laboratory. Oxford: Oxford Regional Health Authority, 1990.

16 Stott NCH. Desktop laboratory technology in general practice. BMF 1989;229:579-80.

17 Hobbs R. Near patient testing in primary care [Editorial]. BMJ 1996;312:263-4.

18 Anderson JR, Linsell WD, Mitchell FM. Guidelines on the performance of chemical pathology assays outside the laboratory. BMF 1981;282:743.

19 Price CP, Burrin JM, Nattrass M. Extra-laboratory blood glucose measurement: a policy statement. Diabetic Med 1988;5:705-9.

20 Burnett D, Freedman D. Near patient testing: the management issues. Health Services Management 1994;3:10-12. 